\title{
Sesgos cognitivos en la comunicación y prevención de la COVID-19
}

\section{Cognitive biases in communication and prevention of COVID-19}

\author{
Marta Cerezo Prieto. Universidad de Salamanca. España. \\ marta_cp@usal.es \\ $[\mathrm{CV}] \odot \mathrm{G}$
}

El presente trabajo ha sido financiado por la Ayuda para Financiar la Contratación Predoctoral de Personal Investigador de la Junta de Castilla y León y cofinanciado por el Fondo Social Europeo BDNS (Identif.: 376062). Fecha de inicio y término de la presente investigación: 15 de abril de 2020 - 15 de junio de 2020.

\begin{abstract}
Cómo citar este artículo / Referencia normalizada
Cerezo Prieto, M. (2020). Sesgos cognitivos en la comunicación y prevención de la COVID-19. Revista Latina de Comunicación Social, 78, 419-435. https://www.doi.org/10.4185/RLCS-2020$\underline{1483}$
\end{abstract}

\begin{abstract}
RESUMEN
Introducción: Para que los gobiernos promuevan la salud pública de manera efectiva en el contexto de la pandemia del COVID-19, deben de fomentar medidas y restricciones en el comportamiento de sus ciudadanos sin caer en un estado policial. Las ciencias del comportamiento ofrecen una serie de explicaciones, como los sesgos cognitivos, que pueden servir como punto de partida para comprender el no cumplimiento de las medidas sanitarias. Metodología: Para ello, se realiza una revisión de los sesgos cognitivos que irrumpen en el proceso de toma de decisiones, incluyendo el de aversión a la pérdida, el efecto de arrastre, la autopercepción optimista o el de disponibilidad. Asimismo, se exponen algunas medidas como el diseño de comunicaciones o las herramientas nudge para enfrentarse a ellos. Resultados: Pese a los intentos de concientizar a la población mediante campañas de divulgación y educación, es posible incluir otras herramientas que responden a los problemas de atención o de toma de decisiones de la población, basadas en simplificar, enmarcar y adecuar los contenidos de los mensajes al público objetivo, así como la inclusión de elementos que faciliten la adopción de nuevos hábitos. La progresiva vuelta a hábitos que requieren contacto social conlleva que los agentes públicos se reinventen en el proceso de promoción de nuevos estilos de vida, incluyendo la desinfección de manos, el uso de mascarilla o la distancia interpersonal.
\end{abstract}

PALABRAS CLAVE: COVID-19; nudge; España; ciencias de la conducta; psicología social; políticas públicas; arquitectura de elección; sesgos cognitivos.

\begin{abstract}
Introduction: For governments to promote public health effectively in the context of the COVID-19 pandemic, they must encourage measures and restrictions on the behaviour of their citizens without falling into a police state. Behavioural science provides a number of explanations, such as cognitive bias, that can serve as a starting point for understanding non-compliance with health measures. Methodology: To do so, a review of cognitive biases that disrupt the decision-making process is carried out, including loss aversion, carry-over effect, optimistic self-perception or availability.
\end{abstract}


Likewise, some measures such as the design of communications or nudge tools to face them are presented. Results: Despite the attempts to raise awareness through informative and educational campaigns, it is possible to include other tools that respond to the problems of attention or decision making of the population, based on the simplification, framing and adaptation of the contents of the messages to the target audience, as well as the inclusion of elements that facilitate the adoption of new habits. The progressive return to habits that require social contact means that public agents are reinventing themselves in the process of promoting new lifestyles, including hand disinfection, the use of masks or interpersonal distance.

KEYWORDS: COVID-19; nudge; Spain; behavioral sciences; social psychology; public policy; choice architecture; cognitive biases.

\section{CONTENIDO}

1. Introducción. 2. Desarrollo y discusión. 3. Discusión. 4. Conclusiones. 5. Bibliografía

\section{Introducción}

A finales de enero de 2020 los medios de comunicación españoles comenzaron a informar a la población de manera regular sobre la presencia de un coronavirus en China, denominada por aquel entonces "neumonía de Wuhan". Esta se detectó por vez primera en diciembre de 2019, pero no tardó en alarmar a la Organización Mundial de la Salud (OMS), declarando la pandemia mundial el 11 de marzo de 2020. El primer caso confirmado por COVID-19 en España fue el 31 de enero en la isla de La Gomera (RTVE, 2020a). El 13 de febrero se contabilizó la primera víctima por el virus en España. Hasta nuestros días, se ha contabilizado más de 30.000 fallecidos (Centro de Coordinación de Alertas y Emergencias Sanitarias, 2020a). El pasado 14 de marzo el Gobierno decretó el Estado de Alarma en el territorio nacional (Real Decreto 463/2020, 2020), con las consecuentes medidas de contención de la pandemia: limitación de la circulación de la ciudadanía, cierre de negocios o centros educativos y una serie de recomendaciones a nivel individual para evitar la propagación del virus.

Los gobiernos de todo el mundo se han movilizado para controlar y frenar la propagación del virus, pero de nada sirven sus esfuerzos si el comportamiento de la población no se ve sometido a un cambio. Las campañas masivas de salud pública para frenar la pandemia se multiplicaron, especialmente enfatizando aspectos como el lavado de manos, el uso de mascarilla o la distancia entre personas. Además del frenético ritmo de laboratorios de todo el mundo para conseguir una vacuna, las ciencias sociales y del comportamiento están teniendo un papel muy valioso para desacelerar la propagación del virus.

Las ciencias del comportamiento aplicadas a la gestión sanitaria actual pueden ser de gran utilidad a la hora de informar y de modificar el comportamiento de la ciudadanía (Thaler y Sunstein, 2008). Así, pueden servir para crear medidas para controlar la infección y desarrollar nuevos patrones de comportamiento de cara a futuras pandemias (West et al., 2020). A pesar de los grandes esfuerzos que las autoridades están haciendo para formar e informar a los ciudadanos sobre los hábitos que deben asumir en estos momentos de mitigación de la pandemia, es necesario que se tomen otro tipo de medidas que, además, apelen a la motivación colectiva y a una percepción del riesgo acorde a las circunstancias. Según la OMS (2005), ante un brote epidémico, el objetivo principal de la comunicación es mantener la confianza de la población. Por tanto, el reto de las autoridades públicas es doble. Sus mensajes y medidas deben motivar a la ciudadanía para que confíe en unos resultados que no se verán en el corto plazo. En este sentido, el Estado puede centrar sus esfuerzos en la toma de decisiones de la población en pro de un beneficio para la sociedad en general y los ciudadanos en 
particular (Oliver, 2017). Esta actitud no obliga ni impone un comportamiento, la última decisión siempre la tiene la persona y no se infringe su libertad de elección, pero puede auxiliar o asistir ante una toma de decisiones que no siempre es racional (Cerezo, 2020). Por supuesto, no quiere decir que esta sea la única estrategia que se debe asumir en el contexto de la pandemia. Sin embargo, resulta útil como medida que implica un comportamiento socialmente responsable de la ciudadanía sin recurrir a la imposición normativa.

El objetivo de este artículo es dar explicación a la imperfecta toma de decisiones individuales de la población, que implica la puesta en riesgo de la colectividad y revisar una serie de herramientas que, en otros contextos de emergencia sanitaria, han mitigado y reconducido los comportamientos poco saludables de distintos grupos de la población. El punto de partida son los conocimientos hasta la fecha publicados sobre el empleo de las ciencias de la conducta para abordar sesgos cognitivos aplicados a la gestión de la pandemia del SARS-COV-2. Los artículos de revisión tratan de explorar la bibliografía publicada y situarla en perspectiva en torno a un tema (Ramos, Ramos y Romero, 2003), para así dar una visión general de un estado de la cuestión. Para elaborar esta revisión se ha realizado una búsqueda de publicaciones que contenían los términos "sesgos cognitivos", "ciencias conductuales" y "COVID-19" o "pandemia". Al ser un tema de mucha actualidad, el número de publicaciones que abordaban estos temas era aún escaso. Para obtener más información, se accedió a las páginas web institucionales de los centros o institutos de investigación internacionales de ciencias de la conducta. Estas entidades publicaban información regularmente sobre herramientas o estrategias que podían responder a sesgos cognitivos en torno a la pandemia, por lo que se consideró oportuno incluirlas. Además, se consultaron medios de comunicación en línea y recursos elaborados por el Gobierno para obtener información sobre las medidas que este establecía para solventar dichos sesgos. Como resultado de esta revisión, este artículo se compone de dos partes. En la primera, se centra en la teoría de los sesgos cognitivos para explicar el comportamiento imperfecto de la población. La segunda parte aborda el empleo de herramientas nudge y el diseño de comunicaciones en medios para responder a dichos sesgos.

\section{Desarrollo y discusión}

\subsection{Decisiones imperfectas en situaciones críticas}

¿Por qué el ser humano no siempre adopta las medidas correctas para sí mismo y para la sociedad en general aun sabiendo que lo son? Numerosas teorías de los campos de la filosofía, la psicología, neurociencia o la economía han intentado dar respuesta a la imperfección del comportamiento humano. A continuación, se dará respuesta al no siempre correcto comportamiento social frente a la COVID-19 partiendo de las teorías desarrolladas por Tversky y Kahneman (1979), quienes presentaban la racionalidad humana interferida por sesgos conductuales o heurísticos cognitivos. Estos sesgos cognitivos son atajos que emplea el cerebro cuando procesa información, sin embargo pueden entorpecer la toma de decisiones y provocar un comportamiento irracional o incorrecto (Kahneman, 2011). En el contexto de la pandemia, saber detectar estos sesgos y responder con mecanismos que promuevan las conductas socialmente responsables es imprescindible. La incertidumbre en la que estamos hace que nuestro cerebro recurra aún más a estos sesgos para dar respuestas rápidas e impulsivas a decisiones o conductas que debemos evaluar y a las que nunca antes nos habíamos enfrentado. A continuación se presenta una serie de sesgos cognitivos contextualizados en la pandemia y se hace mención a las respuestas diseñadas por las autoridades para mitigarlos. 


\subsubsection{La aversión a la pérdida}

El sesgo de aversión a la pérdida forma parte de la teoría prospectiva (Kahneman y Twersky, 1979) y explica que los humanos le damos más importancia a una pérdida que a una ganancia del mismo valor, es decir, preferimos evitar una pérdida que adquirir una ganancia. Actualmente, este sesgo hace pensar a la población mucho más en lo que están perdiendo con la crisis sanitaria que en lo que están ganando cumpliendo las medidas sanitarias. En respuesta a esto, los responsables de políticas públicas y de salud deben mitigar esa sobrevaloración de las consecuencias negativas o de las perdidas, apelando también a las positivas, dentro del difícil contexto en el que se presentan.

\subsubsection{El efecto de arrastre}

El sesgo anterior conduce al sesgo de efecto de arrastre o bandwagon, consistente en hacer lo que hace la mayoría de personas independientemente de si es lo correcto o no. Por ejemplo, las primeras semanas del estado de alarma estuvieron protagonizadas por ingentes cantidades de personas que acudían al supermercado a comprar mucho más de lo que se necesitaba (20minutos, 2020). Por miedo a quedarse sin productos y ante el comportamiento de pánico de algunas personas, se producía el arrastre y se imitaba dicho comportamiento.

Para mitigar este sesgo se ha de apelar a la racionalidad, siendo esto complicado, pero no imposible en un contexto de pandemia mundial. Aun así, en respuesta a la saturación de los supermercados, las autoridades políticas y los propios empleados aparecieron en varios medios de comunicación nacionales informando que no iba a haber desabastecimiento y apelando a la tranquilidad (El Mundo, 2020). La eficaz respuesta de los supermercados, reponiendo constantemente las estanterías y llamando a la calma, relajó la presión de afluencia en las semanas siguientes.

\subsubsection{Poco valor a las consecuencias del largo plazo}

El ritmo frenético al que se está sometiendo a la población en las tareas del día a día ha provocado que consumamos el tiempo como si se tratara de comida rápida (fast food), esperando consecuencias y resultados de nuestras acciones a muy corto plazo. En el momento en que nuestro comportamiento implique una serie de consecuencias que no se verán hasta el largo plazo, la motivación de ejercerlos disminuye considerablemente (Frederick, Loewenstein, y O'donoghue, 2002). Esto, trasladado al contexto actual, implica que solo tomaremos medidas de protección y contención si vemos resultados a corto plazo. Vemos el futuro más optimista de lo que puede ser, porque percibimos que el tiempo para solucionar los problemas es mayor. Así, se puede llegar a pensar que "por un día que me salte el confinamiento, no pasará nada, hay muchos días para hacerlo bien". El sesgo que envuelve este tipo de comportamientos se conoce como sesgo del presente, con el cual nuestras decisiones y preferencias se centran en disfrutar más del presente que del futuro. El papel de las autoridades, en este caso, radica también en mostrar mensajes esperanzadores en los que se vean resultados a corto plazo de las medidas que se están llevando a cabo.

\subsubsection{La autopercepción optimista}

El sesgo de autopercepción optimista consiste en la creencia de que es poco probable que nos pasen cosas malas. Las personas a menudo sobreestiman la probabilidad de eventos positivos y subestiman la probabilidad de que ocurran eventos negativos en el futuro (Sharot, 2011). Existen varios factores para explicar el optimismo poco realista, incluido el autocontrol percibido y el buen humor (HelwegLarsen y Shepperd, 2001). Este tipo de sesgo se puede observar entre los fumadores ante las advertencias sanitarias. Sienten que no les va a afectar a ellos mismos (porque seguramente solo les 
pase a quienes fuman más que ellos) y se sienten optimistas frente a la amenaza (Strecher, Kreuter y Kobrin, 1995; Arnett, 2000). En la gestión de la enfermedad, este sesgo puede hacernos sentir menos vulnerables que otros colectivos. Por ejemplo, al principio de la pandemia, se decía que afectaba mayormente a personas de edades avanzadas, por lo que todas las personas jóvenes y de mediana edad no se sentían amenazados y, consecuentemente, menospreciaban las medidas de seguridad. Este sesgo se ha visto reducido en los casos en los que un familiar ha caído gravemente enfermo o ha fallecido porque el riesgo se percibe más cercano (Dryhurst et al., 2020). Esto puede ayudar a modelar los mensajes emitidos por los responsables de políticas sanitarias. Las autoridades sanitarias y los medios de comunicación, frente a esto, deben mostrar un mensaje claro. A pesar de que las afecciones más graves, estadísticamente, sean contraídas por personas de riesgo, el hecho de que lo contraiga una persona fuera de este grupo puede ser un gran peligro. Este peligro existe tanto para uno mismos (hay gente joven y de mediana edad que ha fallecido a causa del COVID-19) como para los demás (pueden ser portadores y contagiárselo a una persona vulnerable). Además, deben recurrir a los sentimientos acercando casos de enfermos reales a aquellas personas que perciben menos riesgo. Por ejemplo, apelando a la empatía y para enfrentarse a este sesgo, el Gobierno italiano lanzó una campaña a mediados de marzo que apelaba directamente al miedo y a la responsabilidad individual (El País, 2020). En ella aparecían imágenes de personas intubadas en estado grave en la cama de un hospital, acompañadas con el texto "¿Qué tal las cañas?" "¿Te ha gustado Milán?" "Tu responsabilidad personal hoy podrá determinar la salud física, económica y psicológica del país. Ninguna orden o mandato, solo la más grande forma de consciencia sobre vuestra vida cambiará la suerte de esta horrible enfermedad". Además de incluir la etiqueta "quédate en casa", también empleada en España. En el mes de agosto, el Gobierno peruano lanzó una campaña similar con la etiqueta "El COVID no mata solo. No seamos cómplices" (Presidencia del Consejo de Ministros, 2020). Uno de los peligros de apelar a emociones como el miedo frente a una amenaza puede tener efectos contradictorios. Si las personas no saben gestionar ese mensaje de miedo que apele a la defensa, pueden sentirse más frustradas y vulnerables (Baron, 2000; Loewenstein, Weber, Hsee, y Welch, 2001). En contra, mostrar el peligro de la amenaza puede hacer que la sociedad se comporte de manera más consciente y responsable si esta se siente capaz de enfrentarse a ello (Slovic y Peters, 2006; Bavel et al., 2020).

\subsubsection{El sesgo de la disponibilidad}

Este sesgo consiste en sobreestimar la información que se nos presenta más disponible y accesible, considerando que aquello que se nos viene a la mente es lo más común o importante (Freudenburg, 1993; Tversky y Kahneman, 1974). En este sentido, si las personas tienen la percepción de que la mayoría de la sociedad se salta las normas, puede ser debido a la excesiva exposición de este tipo de información en los medios de comunicación o a que sus conocidos lo hacen. Para evitar emociones negativas o que la gente sienta que su aislamiento y responsabilidad son vulnerados por parte de algunas personas, es importante que también se refuerce que la mayoría de la ciudadanía es responsable y cumple las normas. Por otro lado, si una persona recibe constantemente informaciones sobre conocidos o familiares que han enfermado y están en estado grave, además de sentirse informada solo con cifras de fallecidos, es más probable que esta persona actúe desde el miedo y se sienta más vulnerable.

\subsubsection{El sesgo de confirmación}

Relacionado con el anterior, es la tendencia a buscar e interpretar informaciones que confirman nuestras percepciones o creencias. Así, se le da más importancia a los argumentos que refuerzan nuestras ideas preconcebidas que a aquellos que las contradicen (Jonas et al., 2001). Tenderemos, pues, a ignorar las informaciones que contradigan o vulneren nuestras preconcepciones. En muchas 
circunstancias, ni siquiera nos damos cuenta de que estamos atrapados en un ciclo de confirmación, buscando solo información de apoyo en lugar de refutarla, una tendencia que Kahneman (2011) interpretó como "lo que vemos es todo lo que hay". Esto aparece, por ejemplo, en las noticias falsas o conspiranoicas. Sobre el origen del virus se han oído informaciones de todo tipo, desde que es una creación de un laboratorio hasta que ha sido traído por extraterrestres. Si a esta última teoría le añadimos que el Departamento de Defensa de Estados Unidos difundió imágenes de posibles ovnis surcando el cielo (BBC, 2020), parece más fácil sustentarse a ella. Frente a estas teorías y noticias falsas, tanto sobre la procedencia como sobre los remedios o gestión del virus, es necesario que las autoridades, los medios de comunicación y los líderes de opinión las neutralicen. Por ejemplo, en España, la Policía Nacional ha lanzado una guía contra los bulos y las fake news del coronavirus (Ministerio de Interior, 2020). Radio Televisión Española también ha lanzado la campaña Stop Bulos, que comprueba y desmiente información que recibe sobre el COVID-19 (RTVE, 2020c).

\subsubsection{La buena imagen}

Uno de los sesgos que han aparecido en respuesta a las medidas de control ejercidas por el Estado, es el de statu quo (Samuelson y Zeckhauser, 1988), referido a la preferencia por lo predeterminado que por la alternativa del cambio. Es decir, a no asumir una situación de cambio y, por tanto, seguir actuando de la forma habitual. En el momento de que un agente externo modifique de algún modo la libertad de actuación de una persona, esta responde apelando a la normalidad y libertad que tenía antes. Esto resulta peligroso para la salud pública si además se asocia esta actitud rebelde a un atractivo para los demás, especialmente entre los colectivos de los más jóvenes o de quienes aprovechan la coyuntura política para mostrar su desacuerdo con las medidas tomadas por el Gobierno. Jugando con las limitaciones y fortalezas de este sesgo, se puede recurrir a la imitación de personas consideradas líderes de opinión o gatekeepers y el reproche a aquellas personas o colectivos que incumplan las normas, cambiado el prototipo de alto status a aquellos que cumplen las normas. Esta técnica ha sido empleada por Fernando Simón (director del Centro de Coordinación de Alertas y Emergencias Sanitarias) en una de sus comparecencias del mes de agosto (La Vanguardia, 2020), en la que hacía un llamamiento a los influencers para que promovieran el uso de la mascarilla en sus redes sociales. También ha sido de gran utilidad el empleo de etiquetas como el \#YoMeQuedoEnCasa, que alentaba al público en redes sociales a mostrar su cumplimiento de las medidas de salud pública, creando también un sentimiento de unidad.

En el siguiente apartado se mencionarán una serie de medidas que pueden mitigar la pandemia, tales como el diseño de intervenciones inspiradas en la Teoría nudge y el diseño de campañas de comunicación en medios.

\section{2. ¿Cómo mitigar la pandemia?}

\subsubsection{Diseño de intervenciones: los nudges}

Según Sunstein (Wofford, 2020), el sesgo que más acecha durante la pandemia es el del exceso de optimismo, puesto que las personas normalmente piensan que son más inmunes a los riesgos que los demás, aunque las prisas por obtener unos resultados tangibles y favorables también han influido en la falta de responsabilidad de la población.

Las ciencias de la conducta cuentan con diversas evidencias útiles frente a esta crisis sanitaria. A continuación, se exponen una serie de herramientas que han sido efectivas a nivel internacional o en otros contextos en torno a la higiene individual y el distanciamiento interpersonal. Estas herramientas, en el contexto de los estudios del comportamiento, se conocen como nudges, 
traducidos como pequeños empujones o toques que se aplican para el fomento de hábitos saludables o responsables. El concepto de nudge parte de la teoría de que nuestras decisiones o pensamientos no siempre son racionales y conscientes, sino que interfieren sesgos. Este término se popularizó tras la publicación de Thaler y Sunstein en 2008, donde se definía como "cualquier aspecto de la arquitectura de las decisiones que modifica la conducta de las personas de una manera predecible si prohibir ninguna opción ni cambiar de forma significativa sus incentivos económicos. Para que se pueda considerar como un nudge, debe ser barato y fácil de evitar. Los nudges no son órdenes. Colocar la fruta de forma muy visible es un nudge, prohibir la comida basura no lo es" (Thaler y Sunstein, 2008: 20).

Los nudges basan su funcionalidad en la promoción de comportamientos positivos en la población mediante campañas educativas, modificación de elementos del entorno, etc. De tal suerte, resultan de gran utilidad e interés en el contexto en el que estamos, donde nuestros hábitos tienen que cambiar radicalmente. Tal es su eficacia, que están presentes en informes internacionales que promueven los hábitos en salud pública (OMS, 2012; Institute of Government, 2010; Halpern, 2015).

El empleo de nudges ayuda a motivar comportamientos saludables y socialmente responsables. Favorecen la creación de nuevas rutinas (contribuye a cerrar la brecha entre nuestras intenciones y acciones) y facilita la adquisición de nuevos comportamientos. Esto se consigue con recordatorios y avisos de manera regular sobre las medidas a adoptar (Sunstein, 2014; Aggarwal, Davies y Sullivan, 2016) o convirtiendo el comportamiento en normas sociales (Galle, 2014). A continuación, se mencionan algunos ejemplos que han funcionado en el cambio de los hábitos más representativos frente a la COVID-19.

\subsubsection{La higiene de manos}

El primero de los consejos que se difundieron para mitigar la propagación del virus fue el lavado de manos frecuente con agua y con jabón. ¿Por qué aún no se ha convertido en un hábito si es sencillo de cumplir? Siguiendo las ideas mencionadas en el apartado anterior, uno de los motivos puede ser, simplemente, que es algo a lo que no estamos acostumbrados (sesgo de la disponibilidad). Otros motivos son que la gente de nuestro alrededor tampoco haya adquirido el hábito (por lo que no se produce el efecto de arrastre) o que se da por hecho que "no hemos tocado nada, nos hemos echado gel hace poco, la superficie que he tocado estaba limpia..." por lo que "no estamos contaminados" (autopercepción optimista). Este hábito en muchas sociedades ha sido determinante en la gestión de otras pandemias.

Heijnen y Greenland (2015) y Huis (et al., 2012) han documentado el uso de nudges para motivar este hábito antes de la llegada de la COVID-19. Emplearon mecanismos como simplificar la información e incluir elemento emotivos y gráficos que llamen la atención de la ciudadanía. A continuación se presentan algunos casos de éxito. Por ejemplo, en los colegios de Bangladesh, para fomentar este hábito por parte de los escolares, han promovido una serie de nudges que consiguen llamarles la atención (Dreibelbis et al., 2016). Los lavabos se han decorado con huellas de manos, se ha pintado en el suelo caminos con pisadas que conducen al mismo y, además, en los espejos, se han pegado dibujos con ojos que "vigilan" la actuación de los pequeños. El propio empleo de espejos en los lavabos públicos resulta eficiente al sentir que otras personas están observando nuestro comportamiento, lo que incita a comportarnos de la manera socialmente más aceptada.

Un estudio realizado en Dinamarca (Birnbach et al., 2012) para fomentar el empleo de gel desinfectante en hospitales concluyó que incluir un cartel de color rojo señalando el desinfectante aumentaba la probabilidad de su uso. Culturalmente, en occidente, el color rojo se emplea en las 
señalizaciones que indica atención, advertencia o incluso detención, por lo que este estimulo visual capta nuestra atención rápidamente (Heller y Mielke, 2004; Kuniecki et al., 2015). En este estudio también se analizaba el grado de efectividad de un mensaje que acompañaba a este cartel: "Aquí usamos desinfectante de manos para proteger a su familiar", lo que produjo resultados muy positivos en comparación con colocar un bote de desinfectante transparente en un lugar poco llamativo. Además, si los geles desinfectantes se presentan en un formato de dispensador automático en lugar de un bote que los usuarios deben agarrar, girar y apretar para que salga el fluido, el empleo del mismo también se puede ver incrementado, ya que se facilita en gran medida la tarea de obtención (evitando también el contacto directo de las manos agarrando los botes). Otro nudge, empleado en 2017 en colegios de Estados Unidos para fomentar la higiene de manos de los niños, consistió en incluir en los manillares de las puertas de los baños una pegatina con un dibujo de gérmenes. De este modo, los pequeños inevitablemente lo tocaban al abrir la puerta y eran conscientes de su presencia, haciendo que aumentara la probabilidad de que se lavaran las manos (Mehta, 2017). Por tanto, apelando a nuestro inconsciente y diseñando comunicaciones llamativas se puede promover hábitos como la higiene de manos.

Para aumentar la duración del lavado de manos se pueden crear hábitos en torno a ello, como cantar el cumpleaños feliz dos veces en vez de contar los segundos recomendados (ABC, 2020a). Algo similar se ha propuesto para que los niños se laven los dientes el suficiente tiempo, ya que suele ser una tarea que les resulta aburrida o inútil. Para que estén entretenidos, se han empleado nudges que incluyen un reloj de arena que indica el tiempo que deben estar cepillándoselos o, yendo un paso más allá, existen campañas que, ayudados de una app y un Smartphone o tablet, muestran el cepillado de dientes como un juego, en el que existe un cronómetro y según avanza el tiempo se va apareciendo en la pantalla un personaje animado. Al finalizar el cepillado, el niño gana un premio (una pegatina virtual) (Nudging for good, 2015). Extrapolándolo al hábito de la higiene de manos, especialmente a los más pequeños, sería interesante fomentarlo como un juego o una actividad entretenida y no como una imposición, ayudados de nudges. En el contexto español, las medidas tomadas para fomentar este hábito se han limitado a alentar a la población a realizarlo a través de medios de comunicación (LaSexta, 2020) y a facilitar carteles y vídeos explicativos en su página web (Centro de Coordinación de Alertas y Emergencias Sanitarias, 2020b). Se ha comprobado que las infografías y carteles con menos texto son más efectivos (The Behavioural Insights Team, 2020), por lo que habría que estudiar la calidad y efectividad de los mensajes en España.

\subsubsection{Distanciamiento social}

El propio concepto de distanciamiento social, como mencionan Bavel et al. (2020), puede llegar a agravar el sentimiento de soledad o estrés. Respondiendo al sesgo de status quo y de aversión a la pérdida, la alternativa de cambio ha de ser lo más atractiva posible, no presentada como una pérdida de libertades, sino como una oportunidad para combatir la amenaza del virus. Si nos definimos como seres sociales (Aronson, 2000), en el momento de hablar de aislamiento social las implicaciones psíquicas para nuestro autoconcepto se ven alteradas. El término alternativo que Bavel et al. (2020) proponen es distanciamiento físico, que implícitamente conserva que el concepto de conexión social es posible. Podemos seguir conectados de otras maneras a pesar de estar físicamente separados.

En el contexto de los espacios públicos, además de controlar el aforo, se pueden emplear nudges para recordar dicho distanciamiento. Por ejemplo, en los últimos meses ya se han colocado pegatinas en el suelo con forma de huellas y así facilitar el cálculo de los dos metros de seguridad. Algo similar se puede emplear en los bares, donde recientemente se han producido irregularidades a causa del difícil cálculo de la distancia entre personas. Incluyendo una pegatina en la propia mesa donde se indiquen los centímetros que hay de un lado a otro puede ayudar a los consumidores a comprender cuál es la verdadera distancia a la que están. 
Durante el verano, los medios de comunicación han denunciado reuniones y fiestas de jóvenes sin respetar las medidas de seguridad (especialmente el distanciamiento), lo que ha provocado una disminución de la edad media de los contagiados (ABC, 2020b). Esto es debido a la autopercepción optimista del sector más joven de la sociedad, la imagen de éxito que relacionan con saltarse las normas y al poco valor que le dan a las consecuencias que esta actitud puede provocar en el largo plazo.

Producir un "efecto de arrastre" es una de las estrategias posibles frente a dicha actitud. Mensajes del estilo a "la mayoría de los jóvenes no ha acudido a fiestas este verano", reforzando actitudes positivas. El empleo de normas socialmente compartidas (no necesariamente jurídicas) puede reducir el comportamiento nocivo (Sunstein, 2014).

\subsubsection{Llevar las manos a la cara y uso de mascarilla}

Las ciencias del comportamiento también pueden ayudarnos a asumir nuevos hábitos como el hecho de evitar tocarnos la cara. En concreto, este hábito es más complicado de asumir dada la inconsciencia con la que habitualmente se realiza. Se puede sustituir la acción, por ejemplo, tocándonos la cara con la muñeca o creando barreras que impidan que nos llevemos las manos a la cara, como tener las manos ocupadas con algo, meterlas en los bolsillos, recogerse el pelo para que no moleste o, en lugares públicos, colocar recordatorios. El hábito que se ha procurado establecer por parte de las autoridades sanitarias es el toser o estornudar hacia el codo. El empleo de mascarillas sirve también para impedir el contacto directo entre las manos con la nariz y la boca, por lo que no parece descabellado su uso habitual.

La obligación de llevar mascarilla en todo momento (Centro de Coordinación de Alertas y Emergencias Sanitarias, 2020) ha traído consigo una serie de problemas a la población. Entre otras, el aumento de gasto en los hogares (OCU, 2020), la limitada vida útil que se les da y el propio hecho de incluirla como un accesorio más en el día a día. Al igual que ocurre con el empleo de gel desinfectante, una solución basada en la Teoría nudge (Wofford, 2020) puede ser incluir carteles recordatorios sobre el empleo de la mascarilla o, del mismo modo que con el distanciamiento social, crear comunicaciones sobre lo que la mayoría de personas hacen.

\subsubsection{Diseño de mensajes}

En el proceso de diseño de las campañas de comunicación se puede relativizar también qué parte del mensaje será más o meno relevante. Con el efecto del encuadre o framing (Goffman, 1974; Buelna, 2015), los comunicadores pueden enfatizar (encuadrar) una explicación de los acontecimientos respecto a otra. Dicho de otra manera, la recepción de una información depende del filtro interpretativo y las categorizaciones por medio de las cuales los medios explican los hechos. Por ejemplo, no es lo mismo decir que el $5 \%$ de la población española se ha inmunizado al virus que decir que el $95 \%$ es aún vulnerable a él. La información fuente es la misma, pero la forma de presentarla cambia totalmente. El encuadre positivo (mostrando la información en ganancias y no en pérdidas) está asociado con una menor percepción del daño. Por ejemplo, se puede dar una sensación de libertad incluyendo afirmaciones como "así la población estará libre de contagio", "cuanto antes superemos esta situación, más rápido volveremos a la normalidad" (The Network for Public Health Law, 2020).

Por su parte, el papel de los partidos políticos, sean afines o no al Gobierno, en ocasiones ha provocado brechas en ese sentimiento de unidad en la población española, hablando de un "ellos" y 
un "nosotros" que enfrenta directamente a grupos con ideologías políticas opuestas, cuestionando la gestión sanitaria del país y, por tanto, creando desconfianza en sectores de la población, lo que puede relajar el sentimiento de inseguridad ante la pandemia, creer más noticias falsas o desacreditar a las autoridades sanitarias, apoyándose en las etiquetas y prejuicios. Frente a esta situación de polarización política debemos comprender que la amenaza del virus es igual para unos votantes que otros, que somos todos contra el virus. Los líderes de opinión y los canales de comunicación deben diluir la división política en el contexto de una emergencia sanitaria mundial. El sentido de grupo, de comunidad, de pertenecer a un equipo, ha acercado a la sociedad española a unirse a todas las escalas. La cooperación frente a la competencia, actuando por el bien común es también uno de las características que distinguen a los humanos del resto de animales. En una sociedad democrática, en el largo plazo, el comportamiento de la ciudadanía frente a la pandemia solo continuará de manera voluntaria si los gobiernos se enfocan en la legitimidad. Las personas están más dispuestas a cumplir las normas si creen que estas se aplican de manera legítima (Van Rooij y Fine, 2020). La legitimidad va de la mano de la honestidad, de la transparencia y del acuerdo entre todas las partes.

A continuación se presenta cómo simplificar los contenidos, apelar a emociones y a la unidad de grupo sirven como herramientas básicas en el diseño de comunicaciones institucionales.

Ante los numerosos estímulos e informaciones que se reciben a lo largo del día y dada su racionalidad limitada, el cerebro tiende a crear atajos mentales que los simplifican. Si una información se ofrece de manera compleja, es muy probable que la información percibida sea muy poco útil. Sin embargo, si los mensajes son sencillos y llamativos pueden crear un aumento de su efectividad. Cuando se percibe una amenaza y se está alerta, interiorizar y procesar la información que a diario y a cuentagotas se recibe sobre la pandemia es más costoso. Por eso es necesario que los mensajes de las autoridades sean sencillos, claros y precisos, para que su efecto sea reducir la incertidumbre y dejar de lado información innecesaria o rimbombante. Por ejemplo, en el contexto español, Fernando Simón, el 11 de marzo explicó a todos los españoles en un vídeo en qué consistía la famosa curva de contagio (RTVE, 2020b), simplificó de manera gráfica y didáctica el proceso de trasmisión de la pandemia.

Para concienciar sobre la responsabilidad social, otro ejemplo es un video del diario Washington Post de una simulación de cómo las restricciones de movimiento pueden ayudar a disminuir los contagios representado con bolas de colores (Stevens, 2020). Ante una amenaza invisible, el sesgo de optimismo se hace presente, ya que es complicado enfrentar algo que no podemos percibir físicamente. Para visibilizar esta amenaza, existen campañas de comunicación que tienen como finalidad mostrar visualmente la fácil propagación del virus. Ante un peligro invisible, el estado de alerta disminuye, por lo que es importante hacer visible el proceso de contagio. Existen numerosos ejemplos (Johnson, 2020) en los que el virus se representa con tinta luminiscente y se muestra cómo tocando cualquier elemento, compartiendo espacio, hablando... todos los personajes acaban contagiados.

Por otro lado, ¿qué mensajes son más eficaces, los que apelan a las emociones o los de carácter imparcial? Apelar a sentimientos, positivos o negativos, afecta la forma de percibir los hechos o situaciones. Hace a los mensajes más persuasivos aquellos cuyo contenido apela a la empatía (a sentimientos como la esperanza o la añoranza) son también más recordados a largo plazo (Andreasen, 1995). Pensemos en las campañas publicitarias de Navidad, donde la solidaridad y el mensaje de unión y reunión tienen tanto éxito. Recurrir a narrativas realistas en las que un personaje cuenta su experiencia en primera persona sobre una situación de dolor resulta muy eficaz para impulsar esa empatía y, consecuentemente, hacernos sentir más unidos a los demás (Bakker et al., 2018; Shen, 2010). En el contexto de la COVID-19, mostrar testimonios de colectivos sanitarios o de 
enfermos puede ayudar a mostrar la cara más visceral y humana de la pandemia. También es cierto es que apelar en exceso a las emociones puede restar atención a la información objetiva sobre el peligro (Loewenstein, Weber, Hsee, y Welch, 2001). Por esta razón, además de informar sobre los fallecidos y los contagios, es necesario que se informe sobre las personas curadas. La fijación obsesiva por la famosa curva de fallecidos y de contagiados puede desencadenar en sentimientos tan negativos que se ignoren las cifras positivas. Este es uno de los grandes retos de los comunicadores de la salud, tanto representantes políticos como medios: abordar la pandemia con objetividad tildando tanto las cifras de fallecimientos y contagiados como la de recuperados, manteniendo un equilibro entre el pesimismo y el optimismo. Si en los medios se muestra a diario a jóvenes reuniéndose y saltándose las normas del estado de alarma, esto podría ocasionar que otros lo imiten, especialmente si no se muestra su detención o a las autoridades multándolos. Es necesario hacer énfasis en aquellos ciudadanos que sí cumplen las normas.

Por último, la identidad de grupo es un ingrediente clave para comprender y modificar la conducta. Por ejemplo, en Texas, para evitar la acumulación de basura en las calles, lanzaron una campaña con el eslogan "No te metas con Texas", que consiguió vincular el orgullo por la región con no tirar residuos (Barton y Grüne-Yanoff, 2015). Nuestra cultura independiente, frente a la interdependiente no se ha de pasar por alto. Por ejemplo, las culturas asiáticas o del sur de América tienen un sentimiento más fuerte de comunidad, tanto en el entorno familiar como en el social, mientras que en las comunidades de Europa Occidental o América del Norte el "yo" está más presente, respaldando el individualismo (Triandis, 1995; Berry et al., 2002). Por tanto, dependiendo de nuestras costumbres y nuestra percepción de la sociedad, abordaremos más o menos fácilmente la pandemia. Esto es un reto para los responsables de salud pública, ya que parece evidente que, en una cultura independiente, por el propio interés en el "yo", se opte por beneficios individuales al asumir las medidas. Por tanto, los comunicadores deben comprender que un mensaje como "qué beneficios tendrá para mí el asumir estas normas" será más fácilmente asumido que uno que apele a la unión o colectividad, como "qué puedo hacer yo para que otras personas no se contagien". La responsabilidad colectiva, dentro de este autoconcepto general individualista, se ha de asumir e interiorizar si se quiere engrandecer el concepto de cuidado. Desde la declaración del estado de alarma, el Estado ha lanzado la campaña \#EsteVirusLoParamosUnidos para aunar el esfuerzo de todo el país en la lucha contra el coronavirus e incentivar las medidas de distanciamiento social. Quién le iba a decir al Dúo Dinámico que su tema "Resistiré" sería el himno de un país 32 años después de su lanzamiento (Blanco, 2020).

\section{Discusión}

Mientras no se consiga una vacuna eficaz contra el coronavirus, los actores públicos mundiales deben mitigar en la medida de lo posible su transmisión. A pesar del tiempo transcurrido desde el conocimiento de la llegada del virus, sigue existiendo transmisión comunitaria debido al incorrecto cumplimiento de las medidas sanitarias propuestas. Los sesgos cognitivos suponen un problema a nivel individual y comunitario que se ha de afrontar. Se ha partido de la obra de Tversky y Kahneman (1979) para categorizarlos, explicarlos y contextualizarlos en torno a la pandemia actual. De estos se concluye que las conductas individualistas son uno de los aspectos más peligrosos para combatir el virus. De este modo, el mayor valor a las pérdidas que a las ganancias en la toma de decisiones, la minusvaloración de las consecuencias en el largo plazo y la autopercepción optimista y no vulnerable, implican que la responsabilidad se diluya. Por otro lado, se ha visto que las fuentes informativas, como los líderes de opinión o los medios de comunicación, es crucial para guiar a diferentes sectores de la población hacia un comportamiento responsable. 
Comprender las limitaciones conductuales (sesgos cognitivos) que presenta el ser humano en momentos de toma de decisiones críticas puede delimitar eficientemente las medidas a seguir a la hora de comunicar y persuadir en favor de la salud comunitaria.

Para mitigar estos sesgos, son necesarias medidas de tipo educativo y de concienciación sobre la enfermedad. Un cambio en los hábitos y el estilo de vida no son sencillos de producirse, y menos aún si en ellos actúa la parte inconsciente de nuestro cerebro.

De ello surge que herramientas como los nudges (Thaler y Sunstein, 2008) puedan ayudar o empujar a esta inconsciencia a convertir unas medidas sanitarias en hábitos, creando normas sociales, simplificando las informaciones oficiales o rediseñando elementos del entorno. Por otro lado, el diseño de mensajes en campañas de comunicación de acuerdo a las características socioculturales y al grupo social al que van dirigidas, es esencial para que sean efectivas. Se ha realizado una revisión de estos dos aspectos partiendo de los comportamientos en los que más se ha incidido (Centro de Coordinación de Alertas y Emergencias Sanitarias, 2020b), como son la higiene de manos, la distancia interpersonal y el contacto con la cara.

\section{Conclusiones}

Los documentos revisados corroboran la eficacia de estos elementos a la hora de adquirir nuevos hábitos, bien por su similitud con otros contextos previamente estudiados (Sunstein, 2014; Strecher et al., 1995; Shen, 2010; Samuelson y Zeckhauser, 1988; Nudging for Good, 2015; Dreibelbis et al., 2016), bien por su estudio durante la crisis del coronavirus (Bavel et al., 2020; Goldberg et al., 2020; Wofford, 2020; West et al., 2020; The Network for Public Health Law, 2020; The Behavioural Insights Team, 2020; Stevens, 2020; Johnson, 2020).

Este documento proporciona una revisión de algunas claves de las ciencias del comportamiento que pueden ser empeladas por los responsables de las políticas públicas en salud para mitigar la pandemia de la COVID-19.

Como líneas de investigación futuras se propone recabar nuevos estudios publicados al respecto, asî como hacer un seguimiento de las herramientas empleadas por los actores públicos para concienciar hacia conductas socialmente responsables de la población. Además, se podría extender a contextos internacionales, con el propósito de hacer una comparativa de medidas y analizar su efectividad.

Ante un futuro incierto, la humildad epistémica es una virtud necesaria. Esta se basa en la afirmación de que nuestro conocimiento siempre es provisional e incompleto, y que podría requerir una revisión a la luz de nuevas investigaciones (Angner, 2020). Consideración pertinente para los actores políticos y de salud pública cuando tratan con la realidad en que vivimos y con sus limitaciones.

\section{Bibliografía}

20minutos (11 de marzo de 2020). Compras masivas por el pánico al coronavirus: ¿Por qué el papel higiénico es el producto más demandado? Consultado el 19 de abril de 2020. https://bit.ly/2YSy7PS

ABC (18 de marzo de 2020a). Por qué debes cantar «Cumpleaños Feliz» con tus hijos mientras te lavas las manos. https://bit.ly/2EHWb10 
ABC (12 de agosto de 2020b). La edad media de los casos de Covid baja en la última semana hasta los 36 años. https://bit.ly/31QIPbf

Aggarwal, A., Davies, J., y Sullivan, R. (2016). "Nudge" and the epidemic of missed appointments: Can behavioural policies provide a solution for missed appointments in the health service? Journal of Health Organization \& Management, 30(4), 558-564. https://doi.org/10.1108/JHOM$\underline{\text { 04-2015-0061 }}$

Andreasen, A. R. (1995). Marketing social change: Changing behavior to promote health, social development, and the environment. San Francisco, CA: Jossey-Bass

Angner, E. (2020). Epistemic Humility-Knowing Your Limits in a Pandemic. Behavioral Scientist, Consultado el 13 de mayo de 2020. https://bit.ly/317dRf6

Arnett, J. J. (2000). Optimistic bias in adolescent and adult smokers and nonsmokers. Addictive behaviors, 25(4), 625-632. https://doi.org/10.1016/S0306-4603(99)00072-6

Aronson, E. (2000). El animal social. St. Martin's Press.

Bakker, M. H., van Bommel, M., Kerstholt, J. H. y Giebels, E. (2018). The influence of accountability for the crisis and type of crisis communication on people's behavior, feelings and relationship with the government. Public Relations Review, 44, 277-286. https://doi.org/10.1016/i.pubrev.2018.02.004

Baron, J. (2000). Thinking and deciding. Cambridge University Press.

Barton, A. y Grüne-Yanoff, T. (2015). From libertarian paternalism to nudging and beyond. Review of Philosophy and psychology, 6(3), 341-359. https://doi.org/10.1007/s13164-015-0268-x

Bavel, J.J.V., Baicker, K., Boggio, P.S., Capraro, V., Cichocka, A., Cikara, M., Crockett, M.J., Crum, A.J., Douglas, K.M., Druckman, J.N., Drury, J.N., Dube, O., Ellemers, N., Finkel, E.J., Fowler, J.H., Gelfand, M., Han, S., Haslam, S.A., Jetten, J.,...Willer, R. (2020) Using social and behavioural science to support COVID-19 pandemic response. Nature Human Behaviour. https://doi.org/10.1038/s41562-020-0884-Z

BBC (28 de abril de 2020). Videos del Pentágono: las grabaciones de ovnis que hizo públicas el gobierno de EE.UU. Consultado el 15 de mayo de 2020. https://bbc.in/2QMvN8A

Berry, J. W., Berry, J. W., Poortinga, Y. H., Segall, M. H., \& Dasen, P. R. (2002). Cross-cultural psychology: Research and applications. Cambridge University Press.

Birnbach, D. J., Nevo, I., Barnes, S., Fitzpatrick, M., Rosen, L. F., Everett-Thomas, R. y Arheart, K. L. (2012). Do hospital visitors wash their hands? Assessing the use of alcohol-based hand sanitizer in a hospital lobby. American journal of infection control, 40(4), 340-343. https://doi.org/10.1016/j.ajic.2011.05.006

Blanco, L. (1 de abril de 2020). Dúo Dinámico: “'Resistiré' ya pertenece a los ciudadanos, es el himno oficial de la resistencia al virus". El Mundo. Recuperado de: https://bit.ly/2H2VJej 
Buelna, J.R.S. (2015). Teorías de la comunicación y opinión pública. Razón y palabra, (90), 54. https://www.revistarazonypalabra.org/index.php/ryp/article/view/352

Centro de Coordinación de Alertas y Emergencias Sanitarias (2020a). Actualización no 196. Enfermedad por el coronavirus (COVID-19). Consultado el 31 de agosto de 2020. https://bit.ly/31NL6Uw

Centro de Coordinación de Alertas y Emergencias Sanitarias (2020b). Información para la ciudadanía. Consultado el 30 de agosto de 2020. https://bit.ly/2QMEsYy

Cerezo, M. (2020). El poder del Green Nudge: hacia un comportamiento sostenible de los ciudadanos. En Rivero, R. y Cerezo, M. (coord.). Innovación de las normas ambientales, (pp-31-47). Valencia: Tirant lo Blanch.

Dreibelbis R., Kroeger A., Hossain K., Venkatesh M. y Ram P.K. (2016). Behavior Change without Behavior Change Communication: Nudging handwashing among primary school students in Bangladesh. International journal of environmental research and public health. 13(1), 129. https://doi.org/10.3390/ijerph13010129

Dryhurst, S., Schneider, C.R., Kerr, J., Freeman, A.L., Recchia, G., Van Der Bles, A.M.,Spiegelhalter, D. y Van der Linden, S. (2020). Risk perceptions of COVID-19 around the world. Journal of Risk Research, 1-13. https://doi.org/10.1080/13669877.2020.1758193

El Mundo (10 de marzo de 2020). Ante la alarma en las tiendas, los 'súper' recuerdan: "No somos Venezuela". Consultado el 15 de marzo de 2020. https://bit.ly/3jEII8Z

El País (25 de mayo de 2020). Un anuncio italiano recuerda que "la Covid-19 se combate en hospitales, pero sobre todo afuera". Consultado el 12 de julio de 2020. https://bit.ly/350Ko8q

Frederick, S., Loewenstein, G. y O'donoghue, T. (2002). Time discounting and time preference: A critical review. Journal of economic literature, 40(2), 351-401. https://doi.org/10.1257/002205102320161311

Freudenburg, W.R. (1993). Risk and recreancy: Weber, the division of labor, and the rationality of risk perceptions. Social forces, 71(4), 909-932. https://doi.org/10.2307/2580124

Galle, B. (2014) Tax, Command or Nudge?: Evaluating the New Regulation. Georgetown Law Faculty Publications and Other Works.

Goffman, E. (1974). Frame analysis: An essay on the organization of experience. Harvard University Press.

Goldberg, M.H., Gustafson, A., Maibach, E., Linden, S., Ballew, M.T., Bergquist, P., Kotcher, J., Marlon, J., Rosenthal, S. y Leiserowitz, A. (2020). Social norms motivate COVID-19 preventive behaviors. https://doi.org/10.31234/osf.io/9whp4

Halpern, D. (2015). Inside the nudge unit: How small changes can make a big difference. London, England: WH Allen. 
Heijnen, M. y Greenland, K. (2015). Level of Behaviour Change Achievable by Handwashing with Soap Interventions: A rapid review. Sustainable Sanitation Alliance. Consultado el 15 de junio de 2020. https://bit.ly/384MG61

Heller, E. y Mielke, J. C. (2004). Psicología del color: cómo actúan los colores sobre los sentimientos y la razón. Barcelona: Gustavo Gili.

Helweg-Larsen, M. y Shepperd, J. A. (2001). Do moderators of the optimistic bias affect personal or target risk estimates? A review of the literature. Personality and social psychology review, 5(1), 74-95. https://doi.org/10.1207/S15327957PSPR0501_5

Huis, A., van Achterberg, T., de Bruin, M., Grol, R., Schoonhoven, L. y Hulscher, M. (2012). A systematic review of hand hygiene improvement strategies: a behavioural approach. Implementation Sciences. 7, 92 https://doi.org/10.1186/1748-5908-7-92

Institute for Government (2010). MINDSPACE: Influencing Behaviour through Public Policy. Consultado el 12 de agosto de 2020. https://bit.ly/34Ym5rU

Johnson, L.M. (14 de mayo de 2020). Black light experiment shows how quickly a virus like Covid19 can spread at a restaurant. CNN. https://cnn.it/3lIZmWQ

Jonas, E., Schulz-Hardt, S., Frey, D. y Thelen, N. (2001). Confirmation bias in sequential information search after preliminary decisions: an expansion of dissonance theoretical research on selective exposure to information. Journal of personality and social psychology, 80(4), 557. https://doi.org/10.1037/0022-3514.80.4.557

Kahneman, D. (2011). Thinking Fast and Slow. New York: Farrar, Straus and Giroux.

Kahneman, D. y Tversky, A. (1979). Prospect theory: An analysis of decision under risk. Econometrica, 47, 263-291. https://doi.org/10.2307/1914185

Kuniecki, M., Pilarczyk, J. y Wichary, S. (2015). The color red attracts attention in an emotional context. An ERP study. Frontiers in human neuroscience, 9.

LaSexta (17 de mayo de 2020). Fernando Simón alerta de que "el uso de guantes es peligroso si no se hace muy bien". https://bit.ly/32NdINc

La Vanguardia (20 de agosto de 2020). Fernando Simón anima a los influencers a concienciar sobre el coronavirus. Consultado el 23 de agosto de 2020. https://bit.ly/2ERHgkz

Loewenstein, G. F., Weber, E. U., Hsee, C. K., \& Welch, N. (2001). Risk as feelings. Psychological Bulletin, 127(2), 267-286. https://doi.org/10.1037/0033-2909.127.2.267

Mehta, N. (2017). The Healthy Handwashing Habit - Getting Real with Kids. Nudging for kids. Consultado el 17 de junio de 2020. https://bit.ly/3eEbWTr

Ministerio de Interior (27 de marzo de 2020). La Policía Nacional presenta la primera guía para evitar ser manipulados por las fake news. Madrid. Consultado el 30 de marzo de 2020. https://bit.ly/2Djh0yW 
Nudging for Good (2015). The Magic Timer App - Oral-B. Consultado el 17 de junio de 2020. https://bit.ly/3doukxR

OCU (13 de julio de 2020). Mascarillas para todo y para todos. Consultado el 1 de septiembre de 2020. https://bit.ly/2YXgDBD

Oliver, A. (2017). The Origins of Behavioural Public Policy. Cambridge University Press.

OMS (2005). WHO outbreak communication guidelines (N. WHO/CDS/2005.28). Génova: World Health Organization.

OMS (2012). Communication for Behavioural Impact (COMBI).A toolkit for behavioural and social communication in outbreak response.Luxembourg. WHO Department of Global Capacities Alert and Response. https://bit.ly/3bkDdtd

Presidencia del Consejo de Ministros (30 de agosto de 2020). Gobierno lanza agresiva campaña de comunicación para luchar contra la pandemia "El COVID no mata solo. No seamos cómplices". https://bit.ly/3c9dh48

Ramos, M.H., Ramos M.F. y Romero E. (2003). Cómo escribir un artículo de revisión. Revista de postgrado de la VI Cátedra de Medicina, 126, 1-3.

Real Decreto 463/2020, de 14 de marzo, por el que se declara el estado de alarma para la gestión de la situación de crisis sanitaria ocasionada por el COVID-19. Boletín Oficial del Estado. Madrid, 14 de marzo de 2020, núm. 67, pp. 25390.25400.

RTVE (31 de enero de 2020a). Sanidad Confirma El Primer Caso De Coronavirus En España En La Isla De La Gomera. Consultado el 13 de abril de 2020. https://bit.ly/3jzuhmo

RTVE (11 de marzo de 2020b). Así explica Fernando Simón cuáles son las curvas que puede seguir la epidemia del coronavirus. Consultado el 18 de junio de 2020. https://bit.ly/2CycXOC

RTVE (17 de marzo de 2020c). RTVE verifica los bulos sobre el coronavirus que están circulando en redes sociales. Consultado el 18 de junio de 2020. https://bit.ly/31HuFB6

Samuelson, W. y Zeckhauser, R. (1988). Status quo bias in decision making. Journal of risk and uncertainty, 1(1), 7-59. https://doi.org/10.1007/BF00055564

Sharot, T. (2011). The optimism bias. Current biology, 21(23). https://doi.org/10.1016/j.cub.2011.10.030

Shen, L. (2010). Mitigating psychological reactance: The role of message-induced empathy in persuasion. Human Communication Research, 36, 397-422. https://doi.org/10.1111/j.1468$\underline{2958.2010 .01381 . \mathrm{x}}$

Slovic, P. y Peters, E. (2006). Risk perception and affect. Current directions in psychological science, 15(6), 322-325. ttps://doi.org/10.1111/j.1467-8721.2006.00461.x

Stevens, H. (2020). Por qué brotes como el del coronavirus crecen exponencialmente y cómo 'aplanar la curva'. The Washington Post. Consultado el 18 de junio de 2020. https://wapo.st/319AOhW 
Strecher, V.J., Kreuter, M.W. y Kobrin, S.C. (1995). Do cigarette smokers have unrealistic perceptions of their heart attack, cancer, and stroke risks?. Journal of behavioral medicine, 18(1), 45-54. https://doi.org/10.1007/BF01857704

Sunstein, C. R. (2014). Nudging: A Very Short Guide. Journal of Consumer Policy, 37(4), 583-588. https://doi.org/10.1007/s10603-014-9273-1

Thaler, R. H. y Sunstein, C. R. (2008). Nudge: Improving decisions about health, wealth, and happiness. Penguin.

The Behavioural Insights Team (Marzo de 2020). Testing the efficacy of coronavirus messaging. Consultado el 10 de julio de 2020. https://bit.ly/3gQHXYB

The Network for Public Health Law (2020). Guidance for Framing COVID-19 Messaging Using the Moral Foundations Theory Framework. Consultado el 10 de junio de 2020. https://bit.ly/37SYeJD

Triandis, H.C. (1995). Individualism And Collectivism. (Westview Press).

Tversky, A. y Kahneman, D. (1974). Judgment under uncertainty: Heuristics and biases. Science, 185(4157), 1124-1131. https://doi.org/110.1126/science.185.4157.1124

Van Rooij, B. y Fine, A. (2020). How to Lock Down an Open Society. Behavioral Scientist, Consultado el 10 de mayo de 2020. https://bit.ly/3hSav5C

West, R., Michie, S., Rubin, G.J. y Amlot, R. (2020). Applying principles of behaviour change to reduce SARS-CoV-2 transmission. Natural Human Behaviour. https://doi.org/10.1038/s41562$\underline{020-0887-9}$

Wofford (13 de julio de 2020). Questions for Cass Sunstein: Can We "Nudge" to a Better Pandemic Policy? Washingtonian. Recuperado de https://bit.ly/2DkDPIN

\section{AUTORA:}

\section{Marta Cerezo Prieto}

Investigadora en formación a través de la ayuda para contratación predoctoral de la Junta de Castilla y León, cofinanciada por el Fondo Social Europeo. Es doctoranda en el Programa de Doctorado en Formación en la Sociedad del Conocimiento de la Universidad de Salamanca. Graduada en Comunicación Audiovisual y en posesión del Máster en Investigación en Comunicación Audiovisual por la Universidad de Salamanca, por el que recibió el Premio Extraordinario del Curso 2015-2016. Anteriormente, beneficiaria de dos becas de colaboración en el Servicio de Actividades Culturales de la misma Universidad, de la beca CRUE CePYME del Banco Santander y Técnico en procesamiento y análisis de datos sociopolíticos en el Instituto de Iberoamérica.

Marta_cp@usal.es

Orcid ID: https://orcid.org/0000-0002-4620-8654

Google Scholar: https://scholar.google.es/citations?user=-XbtLhAAAAAJ\&hl=es

ResearchGate: https://www.researchgate.net/profile/Marta_Cerezo_Prieto 